\title{
CALCULATION OF SIZE OF STRUCTURAL CONSTITUENTS OF METAL DEPOSITED BY INDUCTION METHOD WITH APPLICATION OF MECHANICAL VIBRATION
}

\author{
V.S. SENCHISHIN and Ch.V. PULKA \\ Ternopol Ivan Puluj National Technical University, MSEU \\ 56 Russkaya Str., 46001, Ternopol, Ukraine. E-mail: Viktor_Synchyshyn@i.ua
}

\begin{abstract}
Possibility of regulation of structure of deposited metal allows improving service properties of the parts. Present work studies the processes of refining of structure of deposited metal at application of horizontal mechanical vibration. Calculation model for determination of parameters of metal structure of PG-S1 type alloy, deposited by induction method applying mechanical vibration, was developed. Graphic dependencies of carbide constituents in the deposited metal on vibration amplitude and frequency were plotted based on received relationships. 9 Ref., 5 Figures.
\end{abstract}

Key $\boldsymbol{y} \boldsymbol{o} \boldsymbol{r d s}:$ deposited metal, induction surfacing, structure, vibration, simulation

Works [1-3] tried to develop a quantitative theory of effect of parameters of mechanical vibration on solidification of metal melts. The calculations were based on ideas of authors of work [4] on evaluation of breaking of coagulation structures in electrolytes by vibration field. Present work also uses data of these investigations [4] in development of mathematical model for refining of structure of deposited metal of highcarbon chromium PG-S1 type alloy (sormite 1) by vibration field.

Calculation model for evaluation of effect of vibration parameters on structure of deposited metal. Work [5] based on results of experimental investigations showed that application of mechanical vibrations during surfacing can have ambiguous effect on characteristics of deposited metal. Aim of the present work is development of mathematical model, which allows determining optimum values of vibration amplitude and frequency using calculation method. They will provide the best service characteristics of deposited metal. Size of carbide inclusions in deposited metal is taken as optimality criterion.

Let us consider (Figure 1) substrate-metal melt on substrate system, which vibrates along the deposit surface with frequency $\omega$ and amplitude $a$. The thickness of metal melt is regarded as relatively small [5].

Groups of metal particles joined in clusters as a result of coagulation, i.e. adhesion of these particles (see Figure 1), are formed in molten

(c) V.S. SENCHISHIN and Ch.V. PULKA, 2015 metal in surfacing. Particles, representing themselves small clusters $(\geq 0.1 \mu \mathrm{m})$, are joined in larger on size $(\leq 100 \mu \mathrm{m})$. This process takes place in the following way [6]. Molten metal is considered as electrolyte. Forces of different nature (Figure 2) act between the particles of molten metal. Approaching of disperse phase particles promotes for disjoining pressure of metal liquid which is between them. This pressure is determined by molecular attracting forces and electrostatic repulsive forces. Attracting forces are Van der Waals forces, which consist of forces of orientation, induction and dispersion interaction. Repulsive forces appear in overlapping of particle diffusion layers.

Condition of the system is determined by balance of attraction and repulsive energies, which is determined by equation

$$
U=B e^{-k h}-A h^{-2},
$$

where $U$ is the sum energy of particle interaction; $B$ is the multiplier depending on value of electric

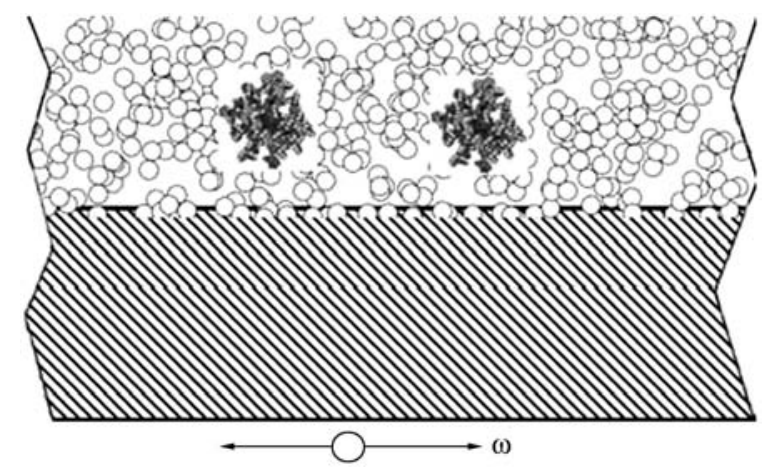

Figure 1. Scheme of formation of clusters in surfacing with vibration 


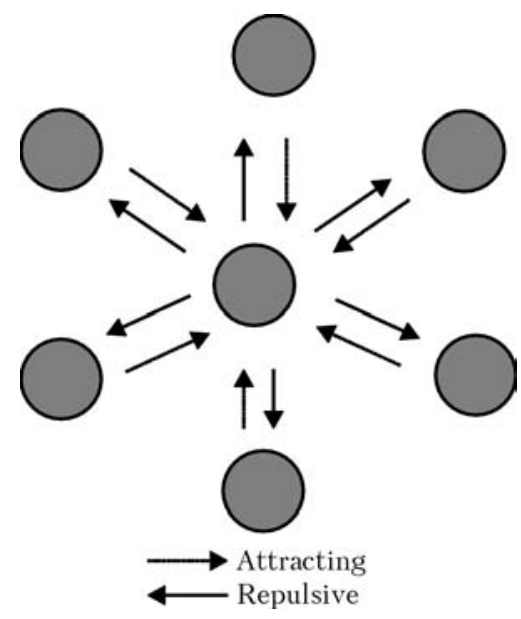

Figure 2. Force action between the particles in metal melt

potentials, properties of environment, and temperature; $k$ is the value inverse to diffusion layer thickness; $h$ is the distance between particles; $A$ is the constant of molecular attracting forces (Hamaker constant). Sum energy $U$ will be negative (secondary potential minimum) at large distances between the particles. Energy $U$ at average distances (around $100 \mu \mathrm{m}$ ) will be positive, that means formation of energy barrier, i.e. electrostatic repulsive forces prevail at this distance. At close distances with prevailing attractive forces, sum energy $U$ of interaction of particles will be positive (primary potential minimum). If energy, which corresponds to potential barrier, is lower than kinetic energy of particles, then they can overcome electrostatic repulsive forces and approach to very small distance (overlapping of double electric layers take place), at which molecular attractive forces prevailing.

Particles are stuck together as a result (i.e. fall in the closest potential well) (Figure 3 ). This deep potential well explains mechanical strength of coagulate. The particles at close distances are bonded due to Van der Waals forces, and formed aggregates acquire some properties of solid body. If energy barrier is high, the particles can not overcome it and develop aggregates.

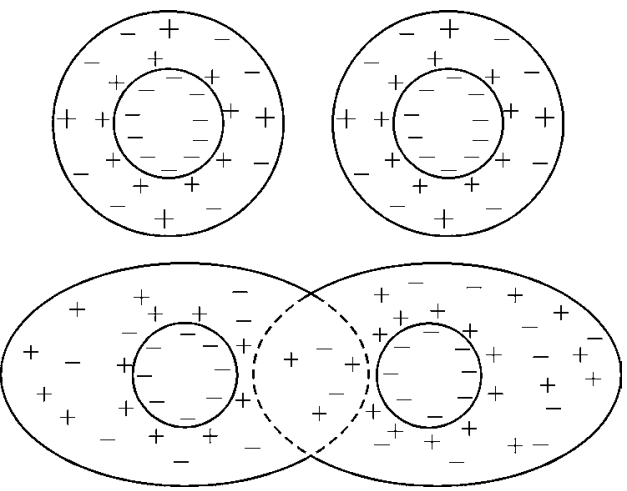

Figure 3. Overlapping of double electric layers and adhesion of particles in melt
Reduction of energy barrier decreases aggregate resistance of the system. For example, adding of electrolyte can reduce thickness of diffusion layer and, thus, decreasing repulsive forces so that energy barrier will escape and particles stick to each other at approaching.

Thus, there are primary and secondary potential wells, depth of which is marked by potential constituents $E_{1}, E_{2}$ and width by $h_{1}$ and $h_{2}$, respectively. Movement of particles of diameter $D$ in the vicinity of each potential maximum is represented as oscillations of harmonic oscillator respectively to own frequencies $f_{1}, f_{2}$, which are found based on solution of Schroedinger equation $[4,7]$ approximated in the following way:

$$
f_{1} \approx \sqrt{E_{1} h_{1}^{-2} m^{-1}}, \quad f_{2} \approx \sqrt{E_{2} h_{2}^{-2} m^{-1}},
$$

where $E_{1}, E_{2}$ values are determined based on [4] in such a way:

$$
\begin{gathered}
E_{1}=n B D\left[0.04 h_{1}^{-1}-0.5 A_{0}\left(1-\ln \left(12 A_{0} h_{1} A^{-1}\right)\right)\right], \\
E_{2}=n B D h_{2}^{-2}\left(0.5-\lambda^{-1} h_{2}^{-1}\right), \\
\lambda=\sqrt{8 \pi q^{2} N \varepsilon^{-1} T^{-1}} .
\end{gathered}
$$

In this case $A_{0}, A, B$ are the constants which depend on system properties [4]; $n$ is the number of particles in structure which are close to the considered; $N$ is the electrolyte concentration (molten metal); $T$ is the temperature in energy units; $q$ is the charge of electrolyte ions; $\varepsilon$ is the dielectric constant; $m$ is the particle mass.

Under static conditions criterion of structure stability will be determined [4] by such inequality:

$$
\begin{gathered}
E_{1}>0.5\left(\rho_{0}-\rho\right) D^{3} g h_{1}, \\
E_{2}>0.5\left(\rho_{0}-\rho\right) D^{3} g h_{2}, \\
E_{1}>\beta T, \quad E_{2}>\beta T .
\end{gathered}
$$

Here $\rho_{0}, \rho$ are the density of dendrite and molten metal, respectively; $g$ is the gravitation acceleration; $\beta \approx 1$.

Let us consider effect of vibration on structure of molten metal, when dendrites of larger size $H$ are formed in it from average size clusters, that in given case corresponds to inequality

$$
H^{2}>>\eta \omega^{-1} \rho^{-1},
$$

where $\eta$ is the average dynamic toughness of molten metal.

Such big aggregates are not going to oscillate in molten metal. Further, oscillations of melt with amplitude $a$ and frequency $\omega$ are recoded. At that, rate $\dot{x}(t)$ of oscillation of melt along the deposit surface on coordinate $x$ can be written in the following way: 


$$
\dot{x}(t)=a \omega \sin \omega t .
$$

Then power $P$, acting on such moving particle of diameter $D$, will be determined according to $[4,8]$ in such a way:

$$
P \approx \eta D^{2} h_{i}^{-1} a \omega .
$$

Taking into account mentioned above, equation of forced vibrations of studied dendrite particle is written respectively in the primary potential well (primary coagulation) under effect of vibration and external force in such a form [4, 7]:

$$
\ddot{x}+2 \xi \dot{x}+f_{1}^{2} x=P m^{-1} \sin \omega t .
$$

In this case $\xi \approx n \eta D^{2} m^{-1} h_{1}^{-1}$.

Linear differential equation of the second order with constant coefficients (9) is solved via representation of the required function $x(t)$ in trigonometric form. As a result the next law of particle oscillation in dendrite volume is received:

$$
x(t)=V_{0} \sin \left(\omega t+\alpha_{0}\right),
$$

where it is formally taken that initial phase $\alpha_{0}$ equals zero and amplitude $V_{0}$ of forced oscillations of the particle equals

$$
\left.V_{0}^{2}=P^{2} m^{-2}\left[\omega^{2}-f_{1}^{2}\right)^{2}+4 \xi^{2} \omega^{2}\right]^{-1} .
$$

If particle of diameter $D$ jumps out from the potential well in vibration, than physically it means that dendrite consisting of such particles will start to collapse. It is possible only if amplitude $V_{0}$ of its oscillations is larger than width of the primary potential well $h_{1}$, i.e. it comes out of the limits of effect of surface force, or receive such energy, which exceeds depth of potential well $E_{1}$, and moves as in absence of surface forces. Based on relationships (3), (8) and (11) these conditions can be written mathematically in such a form:

$$
\begin{gathered}
V_{0} \geq h_{1}, \\
m V_{0}^{2} \omega^{2} \geq E_{1} .
\end{gathered}
$$

$P$ and $V_{0}$ are represented according to (8), (11) in (12), (13), as a result of what the following is received:

$$
\begin{gathered}
\omega^{4}-\omega^{2}\left(2 f_{1}^{2}-4 \xi^{2}+\eta^{2} D^{4} m^{-2} a^{2} h_{1}^{-4}\right)+f_{1}^{4} \leq 0 \\
\left(E_{1}^{-1} \eta^{2} m^{-1} D^{4} h_{1}^{-2} a^{2}-1\right) \omega^{4}+ \\
+\left(2 f_{1}^{2}-4 \xi^{2}\right) \omega^{2}-f_{1}^{4} \geq 0
\end{gathered}
$$

Solution of inequality (14), (15), taking into account (2) and assuming that $m \approx 0.5 \rho D^{3}$ and $\omega$ is always constant, promotes receiving of the following relationships for evaluation of vibration parameters for earlier selected values of diameter $D$ of the particles for which dendrite is decomposed:

$$
\begin{gathered}
\omega^{2} \leq 0.5\left(2 f_{1}^{2}-4 \xi^{2}+4 \rho^{-2} D^{-2} \eta^{2} a^{2} h_{1}^{-4}\right)+ \\
+\sqrt{0.25\left(2 f_{1}^{2}-4 \xi^{2}+4 \rho^{-2} D^{-2} \eta^{2} a^{2} h_{1}^{-4}\right)^{2}-}-\overline{f_{1}^{4}} ; \\
a^{2} \geq \frac{f_{1} \rho^{2} D^{2} h_{1}^{4}\left[\omega^{4}-\left(2 f_{1}^{2}-4 \xi^{2}\right) \omega^{2}+f_{1}^{4}\right]}{4 \eta \omega^{4}} .
\end{gathered}
$$

Thus, if characteristics of molten metal $f_{1}, g$, $\rho, \eta, h_{1}$ are set, than corresponding parameters of vibration $\omega$ and $a$ based on relationships (16) can be found for set grain size $D$ of structure of deposited metal.

Calculation of parameters of structure of deposited metal. Investigations of structure of metal deposited by induction method without and with application of vibrations [5] were carried out for verification of correctness of stated model.

Flat specimens from steel St3 were deposited (Figure 4) with horizontal vibration and without it for performance of investigations by induction method using charge containing powder of PG-S1 alloy. The surfacing was carried out using highfrequency generator of $\mathrm{VChG}-60 / 0.44$ type at constant specific power $W$ and time of surfacing $t$. The modes were similar for two surfacing variants, i.e. anode voltage $10 \mathrm{kV}$; circuit voltage $5.4 \mathrm{kV}$; current of lamp grid $2.2 \mathrm{~A}$; lamp anode current $2 \mathrm{~A}$; surfacing time $35 \mathrm{~s}$; oscillating amplitude $0.2 \mathrm{~mm}$ at $50 \mathrm{~Hz}$ frequency.

Electrolytic method (etching in $20 \%$ solution of chromium acid, voltage $20 \mathrm{~V}$ and holding time $10 \mathrm{~s}$ ) was used for determination of structure of deposited metal. Structure of the base metal was discovered by chemical etching in $4 \%$ solution of nitrogen acid. It is determined that carbides in metal deposited without vibration have 10-

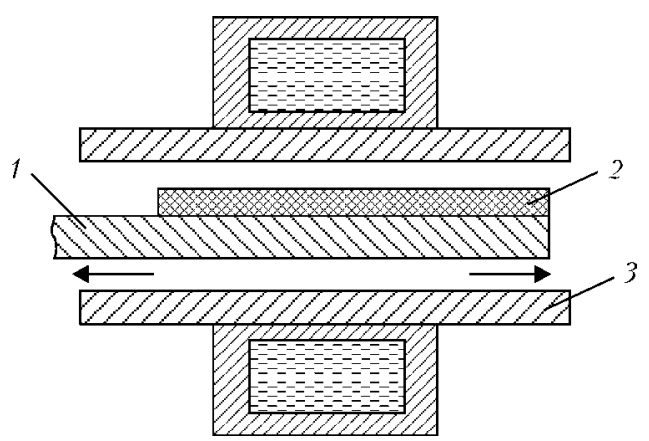

Figure 4. Scheme of induction surfacing: 1 - deposited part; 2 - powdered charge; 3 - inductor (arrows show direction of horizontal vibration) 

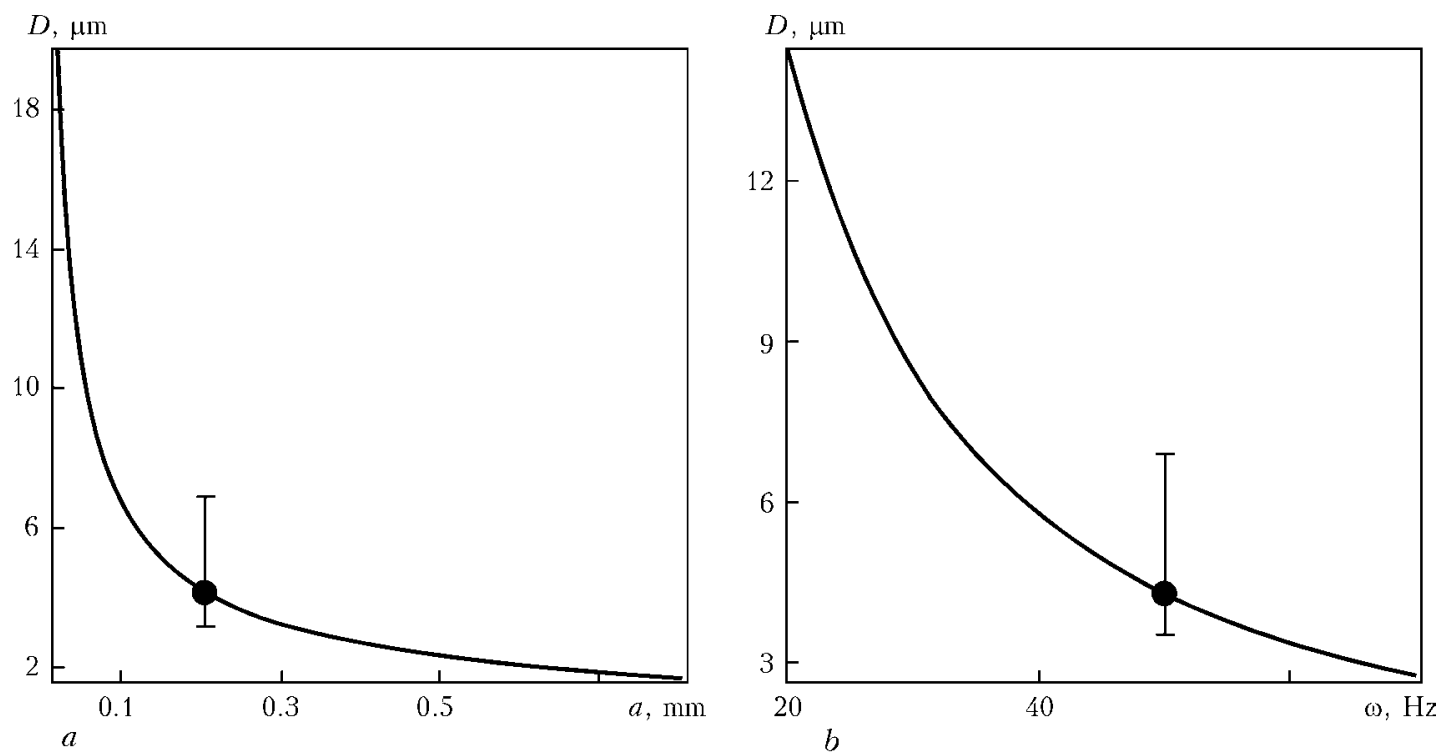

Figure 5. Dependencies of carbide size $D$ on amplitude $a(a)$ and frequency $\omega(b)$ of vibration

$12 \mu \mathrm{m}$ size, and if horizontal vibrations are applied they are refined to $3.5-7.0 \mu \mathrm{m}$ size.

The results were compared with calculation ones by model given above. The following approximated averaged values of characteristics in relationships (16) are given for this purpose for highchromium alloy based on reference data [4, 9]:

$$
\begin{gathered}
\rho=7.8 \cdot 10^{-6} \frac{\mathrm{kg}}{\mathrm{mm}^{3}} ; \quad \eta \approx 7 \cdot 10^{-6} \frac{\mathrm{kg}}{\mathrm{mm} \cdot \mathrm{s}} ; \\
\omega=60 \mathrm{~Hz} ; \quad f_{1} \approx 2 \cdot 10^{3} D^{-1} \mathrm{~Hz} ; \\
\xi \approx 2 \cdot 10^{6} D^{-1} \mathrm{~Hz} ; \quad h_{1}=2 \cdot 10^{-6} \mathrm{~mm} .
\end{gathered}
$$

Dependencies $D-a$ and $D-\omega$ are plotted for more obvious representation of dependence of size $D$ of carbide inclusions on vibration parameters $a$ and $\omega$. Only second relationship (16) was used for that. Considering that $f_{1}^{4}>\omega^{4}-\left(2 f_{1}^{2}-4 \xi^{2}\right) \omega^{2}$, the second relationship can be represented approximately as

$$
D^{3} \geq \frac{8 \cdot 10^{15} \rho^{2} h_{1}^{4}}{a^{2} \eta^{2} \omega^{4}} .
$$

Equation of dependencies $D-a(\omega=50 \mathrm{~Hz})$ and $D-\omega(a=0.2 \mathrm{~mm})$ based on relationship (18) is written in such a form:

$$
D \approx 1.45 a^{-2 / 3} \mu \mathrm{m}, \quad D \approx 785 \omega^{-4 / 3} \mu \mathrm{m} .
$$

Graphical dependencies $D-a$ and $D-\omega$ are plotted based on relationships (19) (Figure 5). The circle on diagrams mark coordinates, where matching of calculation and received in work [5] values of size of carbides on set vibration parame- ters is observed. This indicates correctness and sufficient accuracy of proposed calculation model. Figure 5 shows that increase of vibration parameters $a$ and $\omega$ promotes for significant reduction of carbide size $D$.

Thus, applying relationships (16) and (17) the previously set values of vibrations parameters $a$ and $\omega$ can be chosen, using which desired structure of deposited metal layer can be produced.

1. Bugaj, Yu.M., Pitulej, L.D., Fedenchuk, D.I. (2000) Mathematical model of sedimentation-vibration equilibrium of armitors of composite picks of roller bit. Metody ta Prylady Kontrolyu Yakosti, 6, 100-102.

2. Kryzhanivsky, E.I., Pitulej, L.D., Fedenchuk, D.I. (2005) Influence of vibration on crystalline structure of bit steel. Nauk. Visnyk NTUNG, 12(3), 26-30.

3. Petryna, Yu.D., Pitulej, L.D., Fedenchuk, D.I. (2005) Influence of reinforced melt vibration on crystalline structure of matrix bit steel. Rozvidka ta Rozrobka Naft. i Gaz. Rodovyshch, 16(3), 15-19.

4. Potanin, A.A., Uriev, N.B. (1988) Condition of fracture of coagulation structure by vibration field and aggregability criterion. Teoret. Osnovy Khim. Tekhnologii, 4, 528-534.

5. Pulka, Ch.V., Shably, O.N., Senchishin, V.S. et al. (2012) Influence of vibration of parts on structure and properties of metal in surfacing. The Paton Welding J., 1, 23-25.

6. Tutorsky, I.A. (2008) Introduction to colloid chemistry. Pt 4: Electric surface properties of dispersion systems. Stability and coagulation of colloid systems. Moscow: MITKhT.

7. Landau, L.D., Lifshits, E.M. (1965) Mechanics. Moscow: Nauka.

8. Deryagin, B.V., Churaev, N.V., Muller, V.M. (1985) Surface forces. Moscow: Nauka.

9. Kabachny, V.I., Osypenko, L.K., Grytsan, L.D. et al. (1999) Physical and colloid chemistry. Kharkiv: Prapor. 